\title{
On Finding a Generalized Lowest Rank Solution to a Linear Semi-definite Feasibility Problem
}

\author{
Chee-Khian Sim ${ }^{1}$ \\ Department of Mathematics \\ University of Portsmouth \\ Lion Gate Building, Lion Terrace \\ Portsmouth PO1 3HF \\ United Kingdom
}

April 3, 2015

${ }^{1}$ Email address: chee-khian.sim@port.ac.uk 


\begin{abstract}
In this note, we generalize the affine rank minimization problem and the vector cardinality minimization problem and show that the resulting generalized problem can be solved by solving a sequence of continuous concave minimization problems. In the case of the vector cardinality minimization problem, we show that it can be solved exactly by solving the continuous concave minimization problem.
\end{abstract}

Keywords. Affine rank minimization problem; Vector cardinality minimization problem; Concave minimization problem. 


\section{Introduction}

The affine rank minimization problem is to find a matrix of the lowest rank that satisfies a given system of linear equality constraints. Such a problem arises when solving problems in diverse fields including system identification and control, collaborative filtering and Euclidean embedding. It is well-known that solving the affine rank minimization problem is NP-hard. In [11], it is shown that if a certain restricted isometry property holds for

the linear constraints, the affine rank minimization problem can be solved by solving a convex optimization problem, namely, the minimization of the nuclear norm over the given affine space. This work has its basis on the work [2], which considers the vector cardinality minimization problem. Previous attempts to solve the affine rank minimization problem include $[3,4]$ in which a heuristic is used to solve the problem. Recently, there are interests in the study of concave minimization problems, which we are considering in this note. For example, in [9], convex maximization problems are considered to solve an optimization problem with a sparsity constraint. [15] is another example.

In this note, we consider the problem of finding a generalized lowest rank solution to a linear semi-definite feasibility problem (LSDFP). We observe that the affine rank minimization problem and the vector cardinality minimization problem are special cases of the problem to find a generalized lowest rank solution to an LSDFP. We define this problem in Section 2. The main result of this paper is also presented in the section, where we show that by solving a sequence of general continuous concave minimization problems, we can find a generalized lowest rank solution to an LSDFP. Similar result as this paper has been obtained in [14] for a different sequence of minimization problems. In Section 3, we show that the vector cardinality minimization problem can be solved exactly by solving the continuous concave minimization problem. Although, similar results have been shown in [12] (see also [5]), in this note, we provide a different and completely new proof of these results. We conclude the note with Section 4. 


\subsection{Notations and Definitions}

The space of symmetric $n \times n$ matrices is denoted by $S^{n}$. The cone of positive semi-

definite (resp., positive definite) symmetric matrices is denoted by $S_{+}^{n}$ (resp., $S_{++}^{n}$ ).

Given a symmetric matrix $X \in S^{n}$, denote its real eigenvalues by $\lambda_{i}(X), i=1, \ldots, n$, with $\lambda_{1}(X) \leq \ldots \leq \lambda_{n}(X)$.

Also, given $X \in S^{n}$, we denote its component at the intersection of the $i^{\text {th }}$ row and the $j^{\text {th }}$ column by $X_{i j}$. In case $X$ is partitioned into blocks of submatrices, then $X_{i j}$ refers to the submatrix in the corresponding $(i, j)$ position.

For $X \in S^{n}, \operatorname{diag}(X)$ stands for a vector in $\Re^{n}$ whose entries are the corresponding main diagonal elements of $X$, while given $x \in \Re^{n}$, $\operatorname{Diag}(x)$ is a matrix in $S^{n}$ with main diagonal entries equal to the corresponding component entries in $x$, with the rest of entries in the matrix equal to zero.

Given $Y \in \Re^{k_{1} \times k_{2}}, \operatorname{rank}(Y)$ refers to the dimension of the column space of $Y$, which is the same as the dimension of the row space of $Y$. In case $Y \in S^{n}$, then $\operatorname{rank}(Y)=$ number of nonzero eigenvalues of $Y$, including multiplicities. $\|Y\|_{F}$ stands for the Frobenius norm of $Y$.

\section{Main Results}

The affine rank minimization problem is to find an $Y^{*} \in \Re^{k_{1} \times k_{2}}$ which solves the following minimization problem:

$$
\begin{array}{ll}
\min & \operatorname{rank}(Y) \\
\text { subject to } & \mathcal{A}(Y)=c, \\
& Y \in \Re^{k_{1} \times k_{2}} .
\end{array}
$$

Here, $\mathcal{A}: \Re^{k_{1} \times k_{2}} \rightarrow \Re^{p}$ is a linear map, and $c \in \Re^{p}$. 
It is known [3] that the affine rank minimization problem can be written as

$$
\begin{array}{ll}
\min & \operatorname{rank}(X)+\operatorname{rank}(Z) \\
\text { subject to } & \mathcal{A}(Y)=c, \\
& \left(\begin{array}{cc}
X & Y \\
Y^{T} & Z
\end{array}\right) \in S_{+}^{k_{1}+k_{2}}
\end{array}
$$

The problem we consider in this paper, which we called the problem of finding a generalized lowest rank solution to an LSDFP, is a generalization of (2), and is to find an $X^{*} \in S^{n}$ that solves

$$
\begin{array}{ll}
\min & \sum_{k=1}^{N} \operatorname{rank}\left(X_{k k}\right) \\
\text { subject to } & \operatorname{Tr}\left(A_{i} X\right)=b_{i}, i=1, \ldots, m, \\
& X \in S_{+}^{n} .
\end{array}
$$

Here, $A_{i} \in S^{n}, i=1, \ldots, m$. Note that $X_{k k} \in S_{+}^{n_{k}}, k=1, \ldots, N$, formed the main block diagonal submatrices of $X \in S_{+}^{n}$. Hence, $\sum_{k=1}^{N} n_{k}=n$.

From now onwards, whenever we consider $X \in S^{n}$ a feasible point of (3), it is partitioned into block submatrices with sizes defined from the set $\left\{n_{k} \mid k=1, \ldots, N\right\}$. For example, the block submatrix at the $(i, j)$ position has size $n_{i} \times n_{j}$.

Let $\mathcal{C}$ be the feasible set of (3). We assume that $\mathcal{C} \neq \emptyset$. It is then clear that there exists an optimal solution to (3).

Let $X^{*}$ be an optimal solution to (3). In the following proposition, we show a geometrical property of an optimal solution to (3) which we will use later in Section 3.

Proposition 2.1 If $X^{*}$ is an optimal solution to (3), then $X^{*}$ is an extreme point of $\mathcal{C}$.

Proof: Suppose to the contrary that $X^{*}$ is not an extreme point of $\mathcal{C}$, then there exist $X_{1}, X_{2} \in \mathcal{C}, X_{1}, X_{2} \neq X^{*}$ with $X^{*}=\beta X_{1}+(1-\beta) X_{2}$ for some $\beta \in(0,1)$. Therefore, we have

$$
\left(X^{*}\right)_{k k}=\beta\left(X_{1}\right)_{k k}+(1-\beta)\left(X_{2}\right)_{k k},
$$

where $1 \leq k \leq N$. 
Since $\left(X_{1}\right)_{k k},\left(X_{2}\right)_{k k}$ are symmetric, positive semidefinite matrices, we have by (4) that $\operatorname{Ker}\left(\left(X^{*}\right)_{k k}\right) \subseteq \operatorname{Ker}\left(\left(X_{1}\right)_{k k}\right)$ and $\operatorname{Ker}\left(\left(X^{*}\right)_{k k}\right) \subseteq \operatorname{Ker}\left(\left(X_{2}\right)_{k k}\right), k=1, \ldots, N$. Since $X^{*}$ is an optimal solution to (3), equalities hold in the above, that is, we must have $\operatorname{Ker}\left(\left(X_{1}\right)_{k k}\right)=\operatorname{Ker}\left(\left(X_{2}\right)_{k k}\right)=\operatorname{Ker}\left(\left(X^{*}\right)_{k k}\right)=d_{k}, k=1, \ldots, N$.

Hence, there exists an orthogonal matrix $Q_{k} \in \Re^{n_{k} \times n_{k}}$ such that each of these matrices is of the form

$$
Q_{k}\left(\begin{array}{cc}
B_{k} & 0 \\
0 & 0
\end{array}\right) Q_{k}^{T},
$$

where $B_{k} \in S_{++}^{n_{k}-d_{k}}$ may be different for each $\left(X^{*}\right)_{k k},\left(X_{1}\right)_{k k}$ and $\left(X_{2}\right)_{k k}, k=1, \ldots, N$. By extending the line containing $X^{*}, X_{1}, X_{2}$ in $S^{n}$ in one of the two directions, we can find an $\hat{X} \in \mathcal{C}$ such that

$$
(\hat{X})_{k k}=Q_{k}\left(\begin{array}{cc}
\hat{B}_{k} & 0 \\
0 & 0
\end{array}\right) Q_{k}^{T},
$$

where $\hat{B}_{k}$ is a symmetric, positive semi-definite matrix in $S^{n_{k}-d_{k}}$, but non-invertible for some $k=1, \ldots, N$, while the rest of $\hat{B}_{k}$ are symmetric, positive semi-definite matrices in $S^{n_{k}-d_{k}}$ which may or may not be invertible. This implies that

$$
\sum_{k=1}^{N} \operatorname{rank}\left((\hat{X})_{k k}\right)<\sum_{k=1}^{N} \operatorname{rank}\left(\left(X^{*}\right)_{k k}\right) .
$$

But this is a contradiction to $X^{*}$ being an optimal solution of (3). Hence, $X^{*}$ is an extreme point of $\mathcal{C}$. QED

We now work towards solving (3) by solving a sequence of continuous concave minimization problems. We consider a general class of concave minimization problems that can be used to solve (3). To do this, let us define a general real-valued function $f:(0,1] \times \Re_{+} \rightarrow \Re$ that is continuous on $(0,1] \times \Re_{+}$, and strictly concave on $\Re_{+}$in the second variable for fixed value of the first variable on $(0,1]$. For ease of presentation, let us denote $f(\alpha, x)$ by $f^{\alpha}(x)$ for $\alpha \in(0,1]$ and $x \in \Re_{+}$.

Let $f^{\alpha}$ have the following properties: 
(a) For each $0<\alpha \leq 1, f^{\alpha}(x) \geq C_{\alpha}$ for all $x \in \Re_{+}$, where $C_{\alpha}$ is a constant. Also, $f^{\alpha}(x) \rightarrow \infty$, as $x \rightarrow \infty$ and $\alpha \rightarrow \alpha_{0}$, where $0<\alpha_{0} \leq 1$.

(b) $f^{\alpha}(0) \rightarrow-\infty$ as $\alpha \rightarrow 0^{+}$. Also, $f^{\alpha}(x)$ is bounded, as $\alpha \rightarrow 0^{+}$and $x \rightarrow x_{0}$, where $x_{0}>0$.

(c) if $g:(0,1] \rightarrow \Re_{+}$is any function such that $g(\alpha) \rightarrow \infty$ as $\alpha \rightarrow 0^{+}$, we have $f^{\alpha}(g(\alpha)) \rightarrow \infty$ as $\alpha \rightarrow 0^{+}$.

(d) if $h:(0,1] \rightarrow \Re_{+}$is any function such that $h(\alpha) \rightarrow 0$ as $\alpha \rightarrow 0^{+}$, we have $f^{\alpha}(h(\alpha))-$ $f^{\alpha}(0) \not \rightarrow-\infty$ as $\alpha \rightarrow 0^{+}$.

Remark 2.1 There exist many functions that satisfy the above conditions. For example, $f^{\alpha}(x)=\log (\beta x+\alpha)$ for $0<\alpha \leq 1$, where $\beta>0$ is fixed, and $f^{\alpha}(x)=-\frac{1}{x+\alpha}+x$ for $0<\alpha \leq 1$ can be checked easily to satisfy the above conditions. We will discuss more about the first function in Subsection 2.1 by relating it to the literature. The motivation for the above properties for $f^{\alpha}$ actually comes from looking at this first function.

Let $F_{k}^{\alpha}: \Re_{+}^{n_{k}} \rightarrow \Re$ be defined by

$$
F_{k}^{\alpha}\left(x_{1}, \ldots, x_{n_{k}}\right):=\sum_{j=1}^{n_{k}} f^{\alpha}\left(x_{j}\right)
$$

$F_{k}^{\alpha}$ is a strictly concave separable function on $\Re_{+}^{n_{k}}$, for each $k=1, \ldots, N, 0<\alpha \leq 1$. $F_{k}^{\alpha}$ is also a permutation-invariant function - the definition of a permutation-invariant function can be found in [7].

We have an associated eigenvalue function $G_{k}^{\alpha}=F_{k}^{\alpha} \circ \lambda$ defined on $S_{+}^{n_{k}}$ by

$$
G_{k}^{\alpha}(Y)=\left(F_{k}^{\alpha} \circ \lambda\right)(Y):=\sum_{j=1}^{n_{k}} f^{\alpha}\left(\lambda_{j}(Y)\right)
$$

where $Y \in S_{+}^{n_{k}}$, and $0 \leq \lambda_{1}(Y) \leq \ldots \leq \lambda_{n_{k}}(Y)$ are the eigenvalues of $Y$. Note that $G_{k}^{\alpha}$ is a continuous function on $S_{+}^{n_{k}}$ since $F_{k}^{\alpha}$ is continuous on $\Re_{+}^{n_{k}}$. See [7] for further properties of an eigenvalue function. $G_{k}^{\alpha}$ is also strictly concave on $S_{+}^{n_{k}}$ since $F_{k}^{\alpha}$ is strictly concave on $\Re_{+}^{n_{k}}$ (see for example, $[8,7]$ ). 
With the above setup, consider the following continuous concave minimization problem:

$$
\begin{array}{ll}
\min & \sum_{k=1}^{N} G_{k}^{\alpha}\left(X_{k k}\right) \\
\text { subject to } & \operatorname{Tr}\left(A_{i} X\right)=b_{i}, i=1, \ldots, m, \\
& X \in S_{+}^{n} .
\end{array}
$$

that we will use to solve (3).

Property (a) of $f^{\alpha}$ ensures existence of optimal solutions to (5) (which is the concave minimization problem we want to consider), while properties (a)-(d) of $f^{\alpha}$ relates (5) to (3), the ultimate problem we wish to solve in this note.

We have the following proposition on (5) before we show that its optimal solution is closely related to the optimal solution to (3):

Proposition 2.2 There exists an optimal solution to (5) for each $\alpha, 0<\alpha \leq 1$.

Proof: The objective function of (5) is bounded from below on $\mathcal{C}$ by property (a) of $f^{\alpha}$. Since $\mathcal{C} \neq \emptyset$, this implies that there exists a sequence $\left\{X_{l}\right\} \subset \mathcal{C}$ such that $\sum_{k=1}^{N} G_{k}^{\alpha}\left(\left(X_{l}\right)_{k k}\right)$ converges to the finite optimal value of $(5)$, as $l \rightarrow \infty$.

We have $\left\{X_{l}\right\}$ is bounded. If not, then given that $X_{l} \in S_{+}^{n}$ for each $l$, we can assume without loss of generality that $\sum_{k=1}^{N} \sum_{j=1}^{n_{k}} \lambda_{j}\left(\left(X_{l}\right)_{k k}\right) \rightarrow \infty$, as $l \rightarrow \infty$. This implies that $\sum_{k=1}^{N} G_{k}^{\alpha}\left(\left(X_{l}\right)_{k k}\right) \rightarrow \infty$, as $l \rightarrow \infty$, by property (a) of $f^{\alpha}$, which is impossible.

Hence, there exists a cluster point $\hat{X}$ of $\left\{X_{l}\right\}$, as $l \rightarrow \infty$. Since $\mathcal{C}$ is closed, $\hat{X} \in \mathcal{C}$ and is in fact an optimal solution to (5). QED

Let $X_{\alpha}^{*}$ be an optimal solution to (5).

Since $\sum_{k=1}^{N} G_{k}^{\alpha}\left(X_{k k}\right)$ is a concave function on $S_{+}^{n}$, we have by Corollary 32.3.1 of [13], an optimal solution to $(5)$ is an extreme point of $\mathcal{C}$. In fact, due to the strict concavity of $\sum_{k=1}^{N} G_{k}^{\alpha}\left(X_{k k}\right)$, all optimal solutions of (5) are extreme points of $\mathcal{C}$.

The following theorem is the main result in this section, where we relate solving the concave minimization problem (5) to finding the generalized lowest rank solution to an LSDFP, that is, solving (3): 
Theorem $2.1\left\{X_{\alpha}^{*} \mid 0<\alpha \leq 1\right\}$ is bounded, and every cluster point of $\left\{X_{\alpha}^{*} \mid 0<\alpha \leq\right.$ $1\}$, as $\alpha \rightarrow 0^{+}$, is an optimal solution to (3).

Proof: Let $X^{*}$ be an optimal solution to (3).

We have, by definition of $X_{\alpha}^{*}$, that

$$
\sum_{k=1}^{N} G_{k}^{\alpha}\left(\left(X^{*}\right)_{k k}\right) \geq \sum_{k=1}^{N} G_{k}^{\alpha}\left(\left(X_{\alpha}^{*}\right)_{k k}\right) .
$$

We now show that every cluster point of $\left\{X_{\alpha}^{*} \mid 0<\alpha \leq 1\right\}$, as $\alpha \rightarrow 0^{+}$, is an optimal solution to (3).

Let the sequence $\left\{\alpha_{l}\right\} \subseteq(0,1]$ with $\alpha_{l} \rightarrow 0^{+}$, as $l \rightarrow \infty$, be such that $X_{\alpha_{l}}^{*}$ converges to $\hat{X}^{*} \in \mathcal{C}$, as $l \rightarrow \infty$. That is, $\hat{X}^{*}$ is a cluster point of $\left\{X_{\alpha}^{*} \mid 0<\alpha \leq 1\right\}$ as $\alpha \rightarrow 0^{+}$.

Let

$$
\begin{aligned}
& N_{k}^{*}=\left\{j \in\left\{1, \ldots, n_{k}\right\} \mid \lambda_{j}\left(\left(X^{*}\right)_{k k}\right)=0, \lambda_{j}\left(\left(X_{\alpha_{l}}^{*}\right)_{k k}\right) \rightarrow 0 \text { as } l \rightarrow \infty\right. \text {, with infinitely } \\
& \text { many } \left.l \text { such that } \lambda_{j}\left(\left(X_{\alpha_{l}}^{*}\right)_{k k}\right)>0\right\} \text {, } \\
& N_{k^{\prime}}^{*}=\left\{j \in\left\{1, \ldots, n_{k}\right\} \mid \lambda_{j}\left(\left(X^{*}\right)_{k k}\right)=0, \lambda_{j}\left(\left(X_{\alpha_{l}}^{*}\right)_{k k}\right)=0 \text { for all } l \text { large enough }\right\}, \\
& B_{k, 1}^{*}=\left\{j \in\left\{1, \ldots, n_{k}\right\} \mid \lambda_{j}\left(\left(X^{*}\right)_{k k}\right) \neq 0, \lambda_{j}\left(\left(X_{\alpha_{l}}^{*}\right)_{k k}\right) \rightarrow 0 \text { as } l \rightarrow \infty\right. \text {, with infinitely } \\
& \text { many } \left.l \text { such that } \lambda_{j}\left(\left(X_{\alpha_{l}}^{*}\right)_{k k}\right)>0\right\} \text {, } \\
& B_{k, 1^{\prime}}^{*}=\left\{j \in\left\{1, \ldots, n_{k}\right\} \mid \lambda_{j}\left(\left(X^{*}\right)_{k k}\right) \neq 0, \lambda_{j}\left(\left(X_{\alpha_{l}}^{*}\right)_{k k}\right)=0 \text {, for all } l \text { large enough }\right\}, \\
& B_{k, 2}^{*}=\left\{j \in\left\{1, \ldots, n_{k}\right\} \mid \lambda_{j}\left(\left(X^{*}\right)_{k k}\right)=0, \lambda_{j}\left(\left(X_{\alpha_{l}}^{*}\right)_{k k}\right) \not \rightarrow 0 \text { as } l \rightarrow \infty\right\}, \\
& B_{k, 3}^{*}=\left\{j \in\left\{1, \ldots, n_{k}\right\} \mid \lambda_{j}\left(\left(X^{*}\right)_{k k}\right) \neq 0, \lambda_{j}\left(\left(X_{\alpha_{l}}^{*}\right)_{k k}\right) \not \rightarrow 0 \text { as } l \rightarrow \infty\right\} .
\end{aligned}
$$

It is clear that the above sets are disjoint, and $\left|N_{k}^{*}\right|+\left|N_{k^{\prime}}^{*}\right|+\left|B_{k, 1^{\prime}}^{*}\right|+\sum_{i=1}^{3}\left|B_{k, i}^{*}\right|=n_{k}$, for $k=1, \ldots, N$.

Note that $\operatorname{rank}\left(\left(\hat{X}^{*}\right)_{k k}\right)=\left|B_{k, 2}^{*}\right|+\left|B_{k, 3}^{*}\right|$ and $\operatorname{rank}\left(\left(X^{*}\right)_{k k}\right)=\left|B_{k, 1}^{*}\right|+\left|B_{k, 1^{\prime}}^{*}\right|+\left|B_{k, 3}^{*}\right|$.

We therefore have

$$
\sum_{k=1}^{N}\left[\left|B_{k, 2}^{*}\right|-\left|B_{k, 1^{\prime}}^{*}\right|-\left|B_{k, 1}^{*}\right|\right] \geq 0
$$

since $X^{*}$ is an optimal solution to (3).

We now claim that (7) is actually an equality. 
If not, then

$$
\sum_{k=1}^{N}\left[\left|B_{k, 2}^{*}\right|-\left|B_{k, 1^{\prime}}^{*}\right|-\left|B_{k, 1}^{*}\right|\right] \geq 1
$$

In the following argument, we consider a subsequence of $\left\{\alpha_{l}\right\}$ if necessary.

Writing (6) using definitions of $G_{k}^{\alpha}$ and the above sets, we have

$$
\begin{aligned}
& \sum_{k=1}^{N}\left[\left(\left|N_{k}^{*}\right|+\left|N_{k^{\prime}}^{*}\right|+\left|B_{k, 2}^{*}\right|\right) f^{\alpha_{l}}(0)+\sum_{j \in B_{k, 1}^{*} \cup B_{k, 1^{\prime}}^{*} \cup B_{k, 3}^{*}} f^{\alpha_{l}}\left(\lambda_{j}\left(\left(X^{*}\right)_{k k}\right)\right)\right] \\
\geq & \sum_{k=1}^{N}\left[\left(\left|N_{k^{\prime}}^{*}\right|+\left|B_{k, 1^{\prime}}^{*}\right|\right) f^{\alpha_{l}}(0)+\sum_{j \in N_{k}^{*} \cup B_{k, 1}^{*} \cup B_{k, 2}^{*} \cup B_{k, 3}^{*}} f^{\alpha_{l}}\left(\lambda_{j}\left(\left(X_{\alpha_{l}}^{*}\right)_{k k}\right)\right)\right]
\end{aligned}
$$

Rearranging terms in (9), the following inequality holds:

$$
\begin{aligned}
& \sum_{k=1}^{N}\left[\left(\left|B_{k, 2}^{*}\right|-\left|B_{k, 1^{\prime}}^{*}\right|-\left|B_{k, 1}^{*}\right|\right) f^{\alpha_{l}}(0)+\sum_{j \in B_{k, 1}^{*} \cup B_{k, 1^{\prime}}^{*} \cup B_{k, 3}^{*}} f^{\alpha_{l}}\left(\lambda_{j}\left(\left(X^{*}\right)_{k k}\right)\right)\right] \\
\geq & \sum_{k=1}^{N}\left[\sum_{j \in B_{k, 2}^{*}} f^{\alpha_{l}}\left(\lambda_{j}\left(\left(X_{\alpha_{l}}^{*}\right)_{k k}\right)\right)+\sum_{j \in N_{k}^{*} \cup B_{k, 1}^{*}}\left(f^{\alpha_{l}}\left(\lambda_{j}\left(\left(X_{\alpha_{l}}^{*}\right)_{k k}\right)\right)-f^{\alpha_{l}}(0)\right)\right. \\
& \left.+\sum_{j \in B_{k, 3}^{*}} f^{\alpha_{l}}\left(\lambda_{j}\left(\left(X_{\alpha_{l}}^{*}\right)_{k k}\right)\right)\right] .
\end{aligned}
$$

We have in (10), its right-hand side does not tend to $-\infty$ as $l \rightarrow \infty$, using properties (b), (c) and (d) of $f^{\alpha_{l}}$. However, since $\lim _{l \rightarrow \infty} f^{\alpha_{l}}(0)=-\infty$ and from (8), we have the left-hand side of (10) tends to $-\infty$ as $l \rightarrow \infty$. But this is a contradiction. Hence, we have

$$
\sum_{k=1}^{N}\left[\left|B_{k, 2}^{*}\right|-\left|B_{k, 1^{\prime}}^{*}\right|-\left|B_{k, 1}^{*}\right|\right]=0 .
$$

Thus, $\hat{X}^{*}$ is an optimal solution to (3). Therefore, every cluster point of $\left\{X_{\alpha}^{*} \mid 0<\alpha \leq\right.$ $1\}$, as $\alpha \rightarrow 0^{+}$, is an optimal solution to (3).

We now show that $\left\{X_{\alpha}^{*} \mid 0<\alpha \leq 1\right\}$ is bounded by contradiction.

Suppose $\left\{X_{\alpha}^{*} \mid 0<\alpha \leq 1\right\}$ is unbounded.

Then, there exists a sequence $\left\{\alpha_{l}\right\} \subseteq(0,1]$ with $\alpha_{l} \rightarrow \alpha^{*}, 0 \leq \alpha^{*} \leq 1$, as $l \rightarrow \infty$, such that $\left\|X_{\alpha_{l}}^{*}\right\|_{F} \rightarrow \infty$, as $l \rightarrow \infty$. 
We first consider the case when $\alpha^{*}=0$.

We then have $\lambda_{j}\left(\left(X_{\alpha_{l}}^{*}\right)_{k k}\right) \rightarrow \infty$ for some $j \in B_{k, 2}^{*} \cup B_{k, 3}^{*}, k=1, \ldots, N$. By properties (b), (c) and (d) of $f^{\alpha_{l}},(10)$ is contradicted.

The case when $0<\alpha^{*} \leq 1$ can be considered in a similar manner to arrive at a contradiction to (10), using property (a) of $f^{\alpha_{l}}$.

Hence, $\left\{X_{\alpha}^{*} \mid 0<\alpha \leq 1\right\}$ is bounded. QED

The following corollary follows immediately from the above theorem.

Corollary 2.1 Suppose (3) has an unique solution. Then $X_{\alpha}^{*}$ converges to the unique solution, as $\alpha \rightarrow 0^{+}$.

\subsection{An Example of $f^{\alpha}$}

Consider $f^{\alpha}(x)=\log (\beta x+\alpha)$ for $0<\alpha \leq 1$, where $\beta>0$ is fixed. We know that $f^{\alpha}$ defined this way satisfies the conditions given in Section 2. Hence, the optimal solution obtained using its continuous concave minimization problem has cluster points being optimal solutions to (3), by Theorem 2.1.

Now for $f^{\alpha}(x)=\log (\beta x+\alpha)$, we can write (5) in a neat way as

$$
\begin{array}{ll}
\min & \sum_{k=1}^{N} \log \operatorname{det}\left(\beta X_{k k}+\alpha I\right) \\
\text { subject to } & \operatorname{Tr}\left(A_{i} X\right)=b_{i}, i=1, \ldots, m, \\
& X \in S_{+}^{n},
\end{array}
$$

where in (5), we have

$$
\begin{aligned}
G_{k}^{\alpha}\left(X_{k k}\right) & =\sum_{j=1}^{n_{k}} f^{\alpha}\left(\lambda_{j}\left(X_{k k}\right)\right) \\
& =\sum_{j=1}^{n_{k}} \log \left(\beta \lambda_{j}\left(X_{k k}\right)+\alpha\right) \\
& =\log \prod_{j=1}^{n_{k}}\left(\beta \lambda_{j}\left(X_{k k}\right)+\alpha\right) \\
& =\log \operatorname{det}\left(\beta X_{k k}+\alpha I\right),
\end{aligned}
$$


hence resulting in (12).

In [3], (12) is used in a heuristic to solve the affine rank minimization problem, with $\beta=1$ and $N=2$, and the authors are able to solve examples of the affine rank minimization problem numerically using the heuristic.

It is easy to see by analyzing the specific form of the objective function in (12) that any cluster points of its optimal solution is an optimal solution to (3), as $\alpha$ tends to zero. We generalize this form of the objective function in Section 2 by looking at eigenvalue functions, and come up with a whole class of continuous concave minimization problems that can now be used to solve (3).

\section{A Related Problem}

A closely related problem to (3) is the following problem:

$$
\begin{array}{ll}
\min & \|\operatorname{diag}(X)\|_{0} \\
\text { subject to } & \operatorname{Tr}\left(A_{i} X\right)=b_{i}, i=1, \ldots, m, \\
& X \in S_{+}^{n} .
\end{array}
$$

Here, $\|x\|_{0}$ is the $l_{0}$ norm of $x \in \Re^{n}$, and is defined as the number of nonzero components of $x$.

Note that (13) is a special case of (3) with $N=n$, and $X_{k k}, 1 \leq k \leq N$, are the main diagonal entries of $X$ in (3).

We have (13) is a generalization of its linear counterpart

$$
\begin{array}{ll}
\min & \|x\|_{0} \\
\text { subject to } & B x=b, \\
& x \geq 0
\end{array}
$$

to the space of symmetric matrices.

Consider the following problem, which is called the vector cardinality minimization problem, studied in [2] (see also [6]):

$$
\begin{array}{ll}
\min & \|x\|_{0} \\
\text { subject to } & B x=b .
\end{array}
$$


We have the following proposition relating (14) to (15):

Proposition $3.1\left(\begin{array}{c}\left(x^{+}\right)^{*} \\ \left(x^{-}\right)^{*}\end{array}\right) \in \Re^{2 n}$ is an optimal solution to

$$
\begin{array}{ll}
\min & \left\|x^{+}\right\|_{0}+\left\|x^{-}\right\|_{0} \\
\text { subject to } & (B-B)\left(\begin{array}{c}
x^{+} \\
x^{-}
\end{array}\right)=b, \\
& x^{+}, x^{-} \geq 0
\end{array}
$$

if and only if $x^{*}=\left(x^{+}\right)^{*}-\left(x^{-}\right)^{*}$ is an optimal solution to (15). In this case,

$$
\begin{aligned}
& \left(x^{+}\right)_{i}^{*}=\max \left\{x_{i}^{*}, 0\right\}, \\
& \left(x^{-}\right)_{i}^{*}=-\min \left\{x_{i}^{*}, 0\right\},
\end{aligned}
$$

$i=1, \ldots, n$.

Proof: Suppose $\left(\begin{array}{c}\left(x^{+}\right)^{*} \\ \left(x^{-}\right)^{*}\end{array}\right)$ is an optimal solution to (16). This means that $\left(x^{+}\right)_{i}^{*}>0$ implies that $\left(x^{-}\right)_{i}^{*}=0$, and $\left(x^{-}\right)_{i}^{*}>0$ implies that $\left(x^{+}\right)_{i}^{*}=0$. Hence, (17) holds and $\left\|x^{*}\right\|_{0}=\left\|\left(x^{+}\right)^{*}\right\|_{0}+\left\|\left(x^{-}\right)^{*}\right\|_{0}$.

Suppose $x^{* *}$ is an optimal solution to (15) with $\left\|x^{* *}\right\|_{0}<\left\|x^{*}\right\|_{0}$. Define

$$
\begin{aligned}
& \left(x^{+}\right)_{i}^{* *}=\max \left\{x_{i}^{* *}, 0\right\} \\
& \left(x^{-}\right)_{i}^{* *}=-\min \left\{x_{i}^{* *}, 0\right\}
\end{aligned}
$$

for $i=1, \ldots, n$. Then it is clear that $\left\|x^{* *}\right\|_{0}=\left\|\left(x^{+}\right)^{* *}\right\|_{0}+\left\|\left(x^{-}\right)^{* *}\right\|_{0}$ and also $\left(\begin{array}{c}\left(x^{+}\right)^{* *} \\ \left(x^{-}\right)^{* *}\end{array}\right)$ is feasible to (16). But this is a contradiction to $\left(\begin{array}{c}\left(x^{+}\right)^{*} \\ \left(x^{-}\right)^{*}\end{array}\right) \in \Re^{2 n}$ being an optimal solution to (16), since we have $\left\|\left(x^{+}\right)^{* *}\right\|_{0}+\left\|\left(x^{-}\right)^{* *}\right\|_{0}=\left\|x^{* *}\right\|_{0}<\left\|x^{*}\right\|_{0}=$ $\left\|\left(x^{+}\right)^{*}\right\|_{0}+\left\|\left(x^{-}\right)^{*}\right\|_{0}$. Hence, $x^{*}=\left(x^{+}\right)^{*}-\left(x^{-}\right)^{*}$ is an optimal solution to (15).

The reverse direction can be shown to be true in a similar manner. QED

The above proposition shows that solving (14) is as hard as solving (15).

Using the results in Section 2, we have in the following theorem a way to solve (14) by solving a continuous concave minimization problem: 
Theorem 3.1 In case $A_{i}, 1 \leq i \leq m$, in (13) are diagonal matrices, then $X_{\alpha}^{*}$, which is an optimal solution to (5), where in (5), $N=n$, and $X_{k k}$ are the main diagonal entries of $X$, is also an optimal solution to (13), and hence in this case, to (14), for all $\alpha>0$ small enough.

Proof: Let $X^{*}$ be any cluster point of $\left\{X_{\alpha}^{*}\right\}$, as $\alpha \rightarrow 0^{+}$. By Theorem 2.1, $X^{*}$ is an optimal solution to (13).

Let $X_{\alpha_{l}}^{*} \rightarrow X^{*}$, as $l \rightarrow \infty$, for some sequence $\left\{\alpha_{l}\right\}$ with $\alpha_{l} \rightarrow 0^{+}$, as $l \rightarrow \infty$.

Observe that if $X$ is an extreme point of $\mathcal{C}$, then $\operatorname{diag}(X) \in \Re^{n}$ is an extreme point of the following convex polyhedron in $\Re^{n}$ :

$$
\mathcal{C}^{\prime}=\left\{x \in \Re^{n} \mid \operatorname{Tr}\left(A_{i} \operatorname{Diag}(x)\right)=b_{i}, i=1, \ldots, m, x \geq 0\right\}
$$

By Proposition 2.1 applied to $(13), X^{*}$ is an extreme point of $\mathcal{C}$. Thus $\operatorname{diag}\left(X^{*}\right)$ is an extreme point of $\mathcal{C}^{\prime}$.

Now, $X_{\alpha_{l}}^{*}$ is also an extreme point of $\mathcal{C}$ for each $l$, since it is an optimal solution to (5). This implies that $\operatorname{diag}\left(X_{\alpha_{l}}^{*}\right)$ is an extreme point of $\mathcal{C}^{\prime}$.

Since $X_{\alpha_{l}}^{*} \rightarrow X^{*}$ as $l \rightarrow \infty$, we have $\operatorname{diag}\left(X_{\alpha_{l}}^{*}\right) \rightarrow \operatorname{diag}\left(X^{*}\right)$ as $l \rightarrow \infty$. But since the set of extreme points of $\mathcal{C}^{\prime}$ is finite (by Corollary 19.1.1 of [13]), we must have $\operatorname{diag}\left(X_{\alpha_{l}}^{*}\right)=\operatorname{diag}\left(X^{*}\right)$ for all $l$ large enough. Now, given $X \in \mathcal{C}$, only $\operatorname{diag}(X)$ is needed to determine whether $X$ solves (13), we then have $X_{\alpha_{l}}^{*}$ solves (13) for all $l$ large enough. Since $\left\{X_{\alpha}^{*} \mid 0<\alpha \leq 1\right\}$ is bounded by Theorem 2.1, the theorem is proved. QED

Similar results as Theorem 3.1 have been obtained in [12] (see also [5]). Above, we provide a different proof of these results. Theorem 3.1 tells us that to solve (14), we can solve a continuous concave minimization problem instead.

Remark 3.1 We cannot extend Theorem 3.1 to general rank minimization problem since the assumption that $A_{i}, 1 \leq i \leq m$, are diagonal matrices allows us to say that $X$ is an extreme point of $\mathcal{C}$ implies that $\operatorname{diag}(X)$ is an extreme point of $\mathcal{C}^{\prime}$, where $\mathcal{C}^{\prime}$ is defined in the proof of the theorem. We can then use the finiteness of the number of extreme points of convex polyhedron in $\Re^{n}$ which is the key in proving the theorem. 
Remark 3.2 It turns out that if $f^{\alpha}$ satisfies the conditions in Assumption 2.1 of [15], then it satisfies properties (a)-(d) in this note, except the property that $f^{\alpha}(0) \rightarrow-\infty$ as $\alpha \rightarrow 0^{+}$. Property (a) in this note is satisfied by Assumption 2.1(b) in [15]; part of property (b) in this note is satisfied by Assumption 2.1(b) in [15]; property (c) is satisfied by Assumption 2.1(c) in [15]; while property (d) in this note is satisfied by Assumption 2.1(c) in [15]. Examples 2.3 and 2.6 in [15] also satisfy $f^{\alpha}(0) \rightarrow-\infty$ as $\alpha \rightarrow 0^{+}$and hence they satisfy all properties (a)-(d). Examples 2.3 and 2.6 in [15] can therefore be used as examples of $f^{\alpha}$ in this note. Note that [15] considers the special case of separable functions.

\section{Conclusion}

This note shows that a minimization problem with discrete objective function (3) can be solved exactly by solving a continuous concave minimization problem (5) under certain conditions (Theorem 3.1), and approximated by the continuous concave minimization problem in general (Theorem 2.1 and Corollary 2.1). The results in this note provide an alternative way to solve (3) by looking at a whole class of continuous concave minimization problems. With known examples, Examples 2.3 and 2.6 in [15], belonging to our class of functions $f^{\alpha}$, it is certainly worthwhile to study further our class of continuous concave minimization problems, which is quite simply defined. There are known methods used to solve a continuous concave minimization problem, see for example, $[1,10]$. These methods should be further explored in our context. Another line of research is to linearize the objective function of the continuous concave minimization problem, and solve the general rank minimization problem using an iterative algorithm on the linearized problem, as was done in [15]. Convergence studies will need to be made on the iterative algorithm, and this will likely require further stronger assumptions than properties (a)-(d). 


\section{References}

[1] H. P. Benson, Deterministic algorithms for constrained concave minimization: a unified critical survey, Naval Research Logistics, Vol. 43(1996), pp. 765-795.

[2] E. J. Candes \& T. Tao, Decoding by linear programming, IEEE Transactions on Information Theory, Vol. 51(2005), pp. 4203-4215.

[3] M. Fazel, H. Hindi \& S. P. Boyd, Log-det heuristic for matrix rank minimization with applications to Hankel and Euclidean distance matrices, Proceedings of the American Control Conference, Denver, Colorado, 2003, pp. 2156-2162.

[4] M. Fazel, H. Hindi \& S. P. Boyd, A rank minimization heuristic with application to minimium order system approximation, Proceedings of the American Control Conference, Arlington, VA, 2001, pp. 4734 - 4739.

[5] G. M. Fung \& O. L. Mangasarian, Equivalence of minimal $l_{0}-$ and $l_{p}-$ norm solutions of linear equalities, inequalities and linear programs for sufficiently small $p$, Journal of Optimization Theory and Applications, Vol. 151(2011), pp. 1-10.

[6] D. Ge, X. Jiang \& Y. Ye, A note on the complexity of $L_{p}$ minimization, Mathematical Programming, Series B, Vol. 129(2011), pp. 285-299.

[7] A. S. Lewis, Nonsmooth analysis of eigenvalues, Mathematical Programming, Series A, Vol. 84(1999), pp. 1-24.

[8] A. S. Lewis, Group invariance and convex matrix analysis, SIAM Journal on Matrix Analysis and Applications, Vol. 17(1996), pp. 927-949.

[9] R. Luss \& M. Teboulle, Conditional gradient algorithms for rank-one matrix approximation with a sparsity constraint, SIAM Review, Vol. 55(2013), pp. 65-98.

[10] P. M. Pardalos \& J. B. Rosen, Methods for global concave minimization: a bibliographic survey, SIAM Review, Vol. 28(1986), pp. 367-379. 
[11] B. Recht, M. Fazel \& P. A. Parrilo, Guaranteed minimum-rank solutions of linear matrix equations via nuclear norm minimization, SIAM Review, Vol. 52(2010), pp. 471-501.

[12] F. Rinaldi, F. Schoen \& M. Sciandrone, Concave programming for minimizing the zero-norm over polyhedral sets, Computational Optimization and Applications, Vol. 46(2010), pp. 467-486.

[13] R. T. Rockafellar, Convex Analysis, Princeton Landmarks in Mathematics, Princeton University Press, 1970.

[14] Y.-B. Zhao, An approximation theory of matrix rank minimization and its application to quadratic equations, Linear Algebra and its Applications, Vol. 437(2012), pp. 77-93.

[15] Y.-B. Zhao \& D. Li, Reweighted $l_{1}$-minimization for sparse solutions to underdetermined linear systems, SIAM Journal on Optimization, Vol. 22(2012), pp. 1065-1088. 